\title{
Correlation of Hyperglycemia and Succinate dehydrogenase Activity During Hymenolepiasis in Mice and Treatment With Praziquantel
}

\author{
J Parvathi $^{1}$, K Aruna ${ }^{2}$ \\ ${ }^{1}$ (P.G Department of Zoology, Vivek Vardhini College, Hyderabad, India) \\ ${ }^{2}$ (Retd.Reader and HOD, P.G Department of Zoology, Vivek Vardhini College, Hyderabad, India)
}

\begin{abstract}
Energy metabolism is the chief controlling factor of all living organisms, mostly by the breakdown of glucose. The higher energy demands in a traumatized host directly affects levels of glucose and the enzyme activity involved in its ultimate breakdown. The variations in the carbohydrate levels of host Mus musculus infected with the intestinal helminth Hymenolepis nana and treated with anthelmintic praziquantel, demonstrated histochemically using Periodic acid-schiff reaction, are qualitatively correlated with the corresponding variations of succinate dehydrogenase, a key energy generating enzyme of the Kreb's cycle, visualized by Nitroblue tetrazolium, in the host intestine. These results are also emphasized for the development of prophylactic chemotherapy, especially in the tropical countries, with prevalence of multi-worm infections.
\end{abstract}

Keywords:- Glucose, hymenolepiasis, Nitroblue tetrazolium, Periodic acid-schiff, succinate dehydrogenase

\section{Introduction}

Hymenolepis nana, the common intestinal dwarf tapeworm of mouse and man, especially in the tropics and the subtropics[1], is the only known cestode which can be transmitted directly[2-3]. In hymenolepiasis not only the histology of the host gets traumatized[4], but marked alterations in the tissue substrates like the carbohydrates, proteins and lipids and various enzymes related to their metabolism[5] have been reported.

The carbohydrate metabolism of various vertebrate hosts, infected with helminth parasites has been studied by numerous investigators[6-8]. But information on the reflection of carbohydrate metabolism on the Kreb's cycle enzymes in the helminth infected hosts is meager and scattered. Succinate dehydrogenase(SDH) activity has been studied in rat liver infected with A.ceylanicum and N.braziliensis[9]. The most important activity of SDH is its ability to transfer electrons to the respiratory chain, by catalyzing the formation of fumarate by removal of one hydrogen atom from each $\alpha$-carbon of succinate. SDH in mammalian tissues has high affinity for succinate oxidation and a low affinity for fumarate reduction[10]. In contrast, SDH in parasitic helminths has high affinity for furmarate than succinate [11-12].

The chemotherapy of parasitic diseases is based on the use of compounds which, in suitable dosage, are more damaging to the invading organism than to the host, inhibiting essential metabolic reactions of the pathogenic organism to a greater degree than those of the host[13].

Hence, in the present investigation, the intestinal carbohydrate metabolism in Mus musculus infected with cestode helminth Hymenolepis nana, subsequently treated with the broad-spectrum anthelmintic praziquantel is histochemically demonstrated using Periodic acid-schiff(PAS) reagent. SDH enzyme activity of the three batches of host intestine is also visualized through Nitroblue tetrazolium technique. Further, a qualitative and comprehensive analysis of these substrate-enzyme variations is done to effectively elucidate the intricate host-parasite interactions and the efficacy of praziquantel[14] in reprimanding the parasite induced alterations in the traumatized host.

\section{Materials and methods}

Mus musculus, the murine host was grouped into control, Hymenolepis nana infected (about 100 viable eggs per mouse, by oral route) and praziquantel treated $\left(0.2 \mathrm{ml}\right.$ suspension at $25 \mathrm{mg} / \mathrm{kg}$ body weight, by oral route, on $16^{\text {th }}$ day of infection) batches, maintained under good laboratory practices (GLP) conditions[15]. Adult, 4-5 week old, male hosts weighing 20-25 gm were used, whose intestine was obtained at the terminal stage of experimentation.

For carbohydrate demonstration by Periodic acid-schiff (PAS) reaction, intestine was fixed in $10 \%$ formaldehyde which stabilizes the carbohydrates[16-17]. Paraffin blocks were made of the fixed tissues and $6 \mu$ thick sections microtomed, which were then subjected to PAS test for microscopic visualization of the carbohydrates[18]. The periodic acid oxidizes glycols 
with vicinal $\mathrm{OH}$ groups to two molecules of aldehydes only and no further. These aldehydes are demonstrated by schiff's reagent, which forms intense red addition compounds with the aldehydes thus formed[19]. The carbohydrate content is proportional to the intensity of the colour.

For the enzyme histochemistry of succinate dehydrogenase, unfixed, $5 \mu$ thick cryostat sections were cut at $-35^{\circ} \mathrm{c}$. In the present work the cryostat used was CRYO-CUT II MICROTOME by Reichert-Jung Company, USA. The dehydrogenases of the tricarboxylic cycle are redemonstrable histochemically by the tetrazolium-formazan reduction reaction[20]. The reduction of the colourless tetrazolium salt, by the hydrogen of the substrate, produces an intensely coloured water-insoluble formazan deposit, which can be seen at the site of enzyme activity[21-23]. The procedure followed in this study is the general histochemical staining method for dehydrogenases[24].

The substrate and enzyme stained intestinal slides of the three host batches were then microphotographed at 40x magnification.

\section{Results}

The carbohydrate variations in the intestine of the three host batches are visualized with Periodic acid-schiff reagent in Figs. 1a to 1d. They were found to be more in the infected host batch, than the control batch intestine of Fig.1a, evidenced by the intense red colour, as shown in Figs. $1 \mathrm{~b}$ and 1c. The mechanical trauma inflicted by Hymenolepis nana in the host intestine is also seen as the disrupted tissue in the lumen. The proglottides of the worm can be seen as a ladder-like structure in the lumen of host intestine in Fig.1c. Praziquantel treated intestine in Fig.1d shows carbohydrate intensity nearing the control batch, proving its efficacy.

The nitroblue tetrazolium stained succinate dehydrogenase(SDH) enzyme activity is seen as coloured formazan deposits in Figs. 2a to 2c. The SDH activity in control host intestine is shown in Fig.2a. But the enzyme activity is increased in the infected host batch of Fig.2b, seen as deeply stained section. The treated intestine shows SDH activity comparable to the control section, seen in Fig.2c, thereby reiterating its effectiveness in reprimanding hymenolepiasis.

\section{Discussion}

Glucose metabolism holds a central key position in the intermediary metabolism of carbohydrates, proteins and lipids. Intestine, the habitat of Hymenolepis nana, is the maximally affected host tissue, through pronounced degree of injury and trauma by disrupting the villi, leading to altered physiology of the host substrates, enzymes, and hematology[25]. The increase in colour intensity of infected tissue section of host for carbohydrates is propotional to the increased carbohydrate metabolism. Since the damaged tissue demands increased energy requirements, the pronounced hyperglycemia is the result of the stress in the local damage alarming stimulus[26] and altered enzymology. This is achieved through either glucogenesis or gluconeogenesis.

The ultimate monosaccharide product of carbohydrate, glucose, is metabolized to yield energy required for the body. Glucose, after a series of reactions through glycolysis to produce pyruvic acid, subsequently enters Kreb's cycle generating reduced nucleotides (NADH and FAD) for generating ATP, through electron transport system. Kreb's cycle provides a source of mitochondrial power for the reduction of fumarate to succinate.

In the present work, it is found that the infected host intestine showed an increase in SDH activity. In helminths the succinate formation is bi-directional (Kreb's cycle and reverse Kreb's cycle) which is excreted by the worm into its surroundings. This excreted helminth succinate could then be absorbed and utilized by the host[27]. The absorbed succinate would then be converted to fumarate to ultimately complete the Kreb's cycle via malate and regeneration of oxaloacetate to yield the increased energy demands of the traumatized host intestinal tissue in combating pathogenesis.

The increased carbohydrates in infected intestine of the host also could lead to the formation of more of pyruvate which as acetyl Co A enters the Kreb's cycle moving in the forward direction through the regular Kreb's cycle; after sufficient succinate is accumulated, the Kreb's cycle would proceed in the forward reaction regenerating the oxaloacetate, thus generating more energy to enable the host to meet its required energy demands during pathogenesis with H.nana. Thus, either of the increased interconversions of succinate and fumarate would result in an increased succinate dehydrogenase activity. Hence the present study of these enzymes could be effectively utilized in the future for combating parasitic manifestations, by targeting this particular stage of Kreb's cycle. 
The effects of pathogenic parasites in the organisms could be eradicated or minimized by exploiting the chemotherapeutic effects upon the differences in essential pathways of host and parasite. The property of praziquantel in recouping and restoring the physiological processes is of great interest since praziquantel effectively affects the glucose metabolism of the helminth. It specially inhibits glucose uptake by the helminth[28-29]. This means that the whole carbohydrate metabolism as well as Kreb's cycle are definitely targeted in the parasite by the drug. This is clearly evidenced in the present histochemical study, where praziquantel restores normalcy in the altered carbohydrate and SDH contents of the infected host intestine by removing the worm burden, thereby reducing and eliminating all the stimuli and toxins for the increased energy demands of the infected host. Thus praziquantel is highly effective against Hymenolepis nana which needs only one host and persists through recurrent autoinfections, thereby traumatizing the host further.

\section{Figures}

5.1 Fig.1: Periodic acid-schiff (PAS) stained mouse intestine sections for histochemical demonstration of carbohydrates.

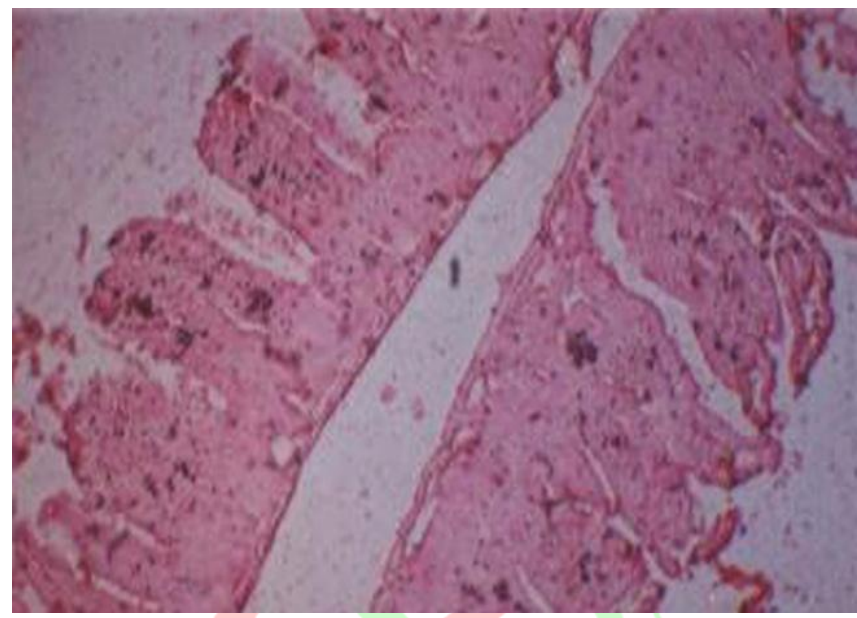

Fig.1a: PAS stained intestine section for carbohydrates, of uninfected control mice showing normal histology.

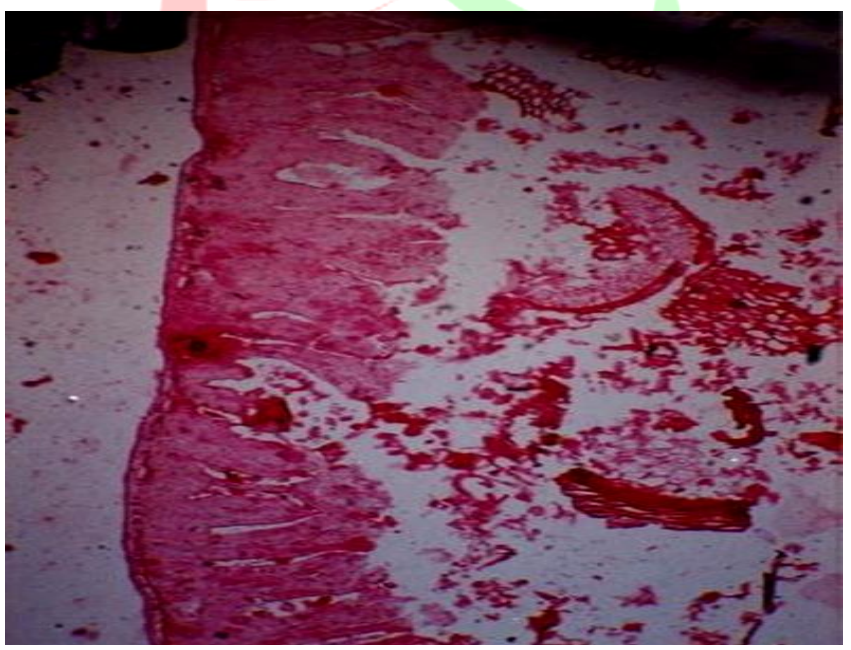

Fig.1b: PAS stained intestine section for carbohydrates, of Hymenolepis nana infected mice showing increased carbohydrate content as increased colour intensity. The mechanical damage inflicted by the worm is seen as debris in the lumen 
IOSR Journal of Pharmacy

Vol. 1, Issue 1, Nov.-Dec, 2012, pp. 022-028

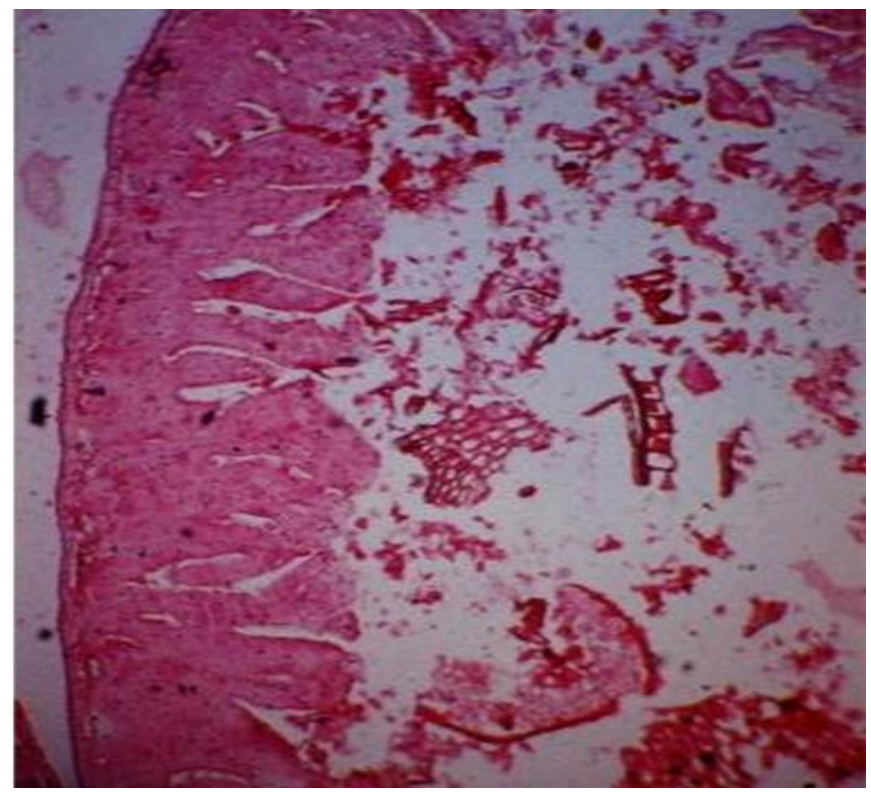

Fig.1c: PAS stained intestine section for carbohydrates, of Hymenolepis nana infected mice showing increased carbohydrate content as increased colour intensity. The proglottides of the worm are seen as the ladder-like structure in the lumen.

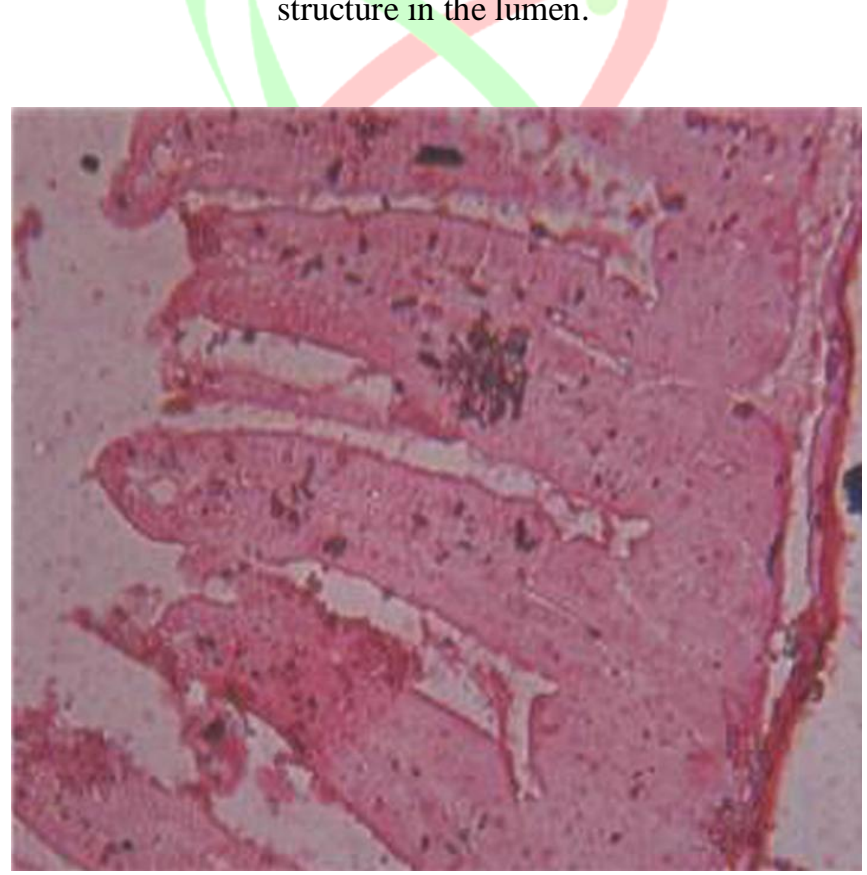

Fig.1d: Periodic acid-schiff stained intestine section for carbohydrates, of Praziquantel treated mice after H.nana infection. The carbohydrate content is near the control intestine, evidenced in its decreased colour intensity over the infected section, proving its efficacy. 
5.2 Fig.2: Nitroblue tetrazolium stained mouse intestine sections for histochemical demonstration of succinate dehydrogenase enzyme activity.

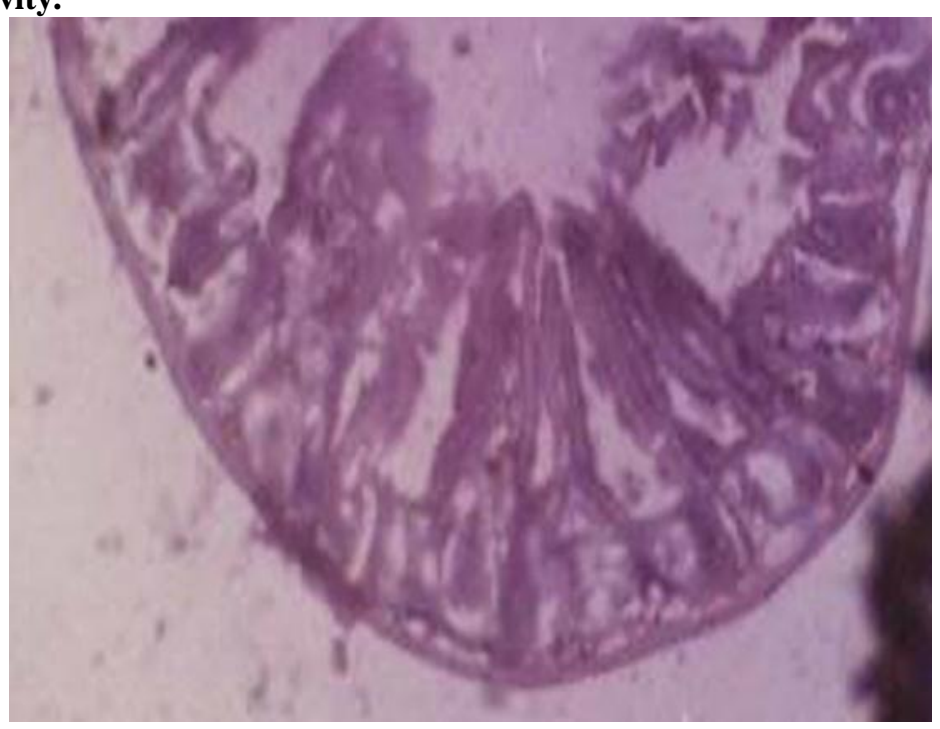

Fig.2a: Nitroblue tetrazolium stained mouse uninfected control intestine section showing succinate dehydrogenase activity.

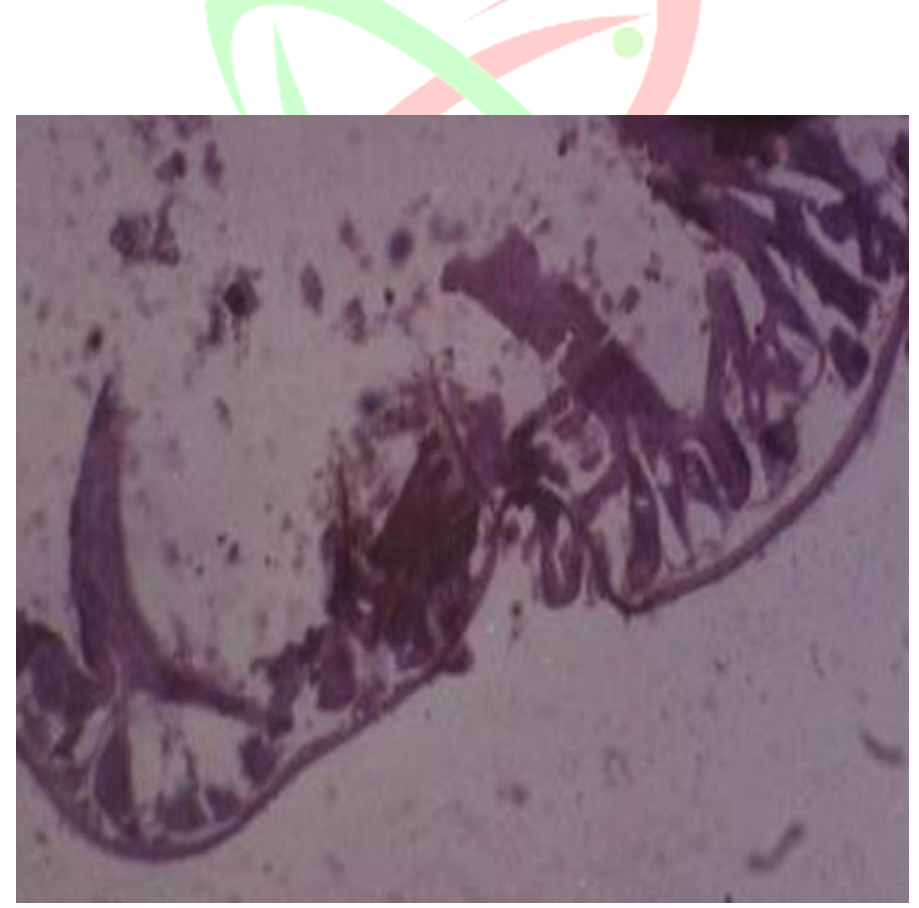

Fig.2b: Nitroblue tetrazolium stained intestine section of mouse infected with Hymenolepis nana, showing increased succinate dehydrogenase activity proportional to the increased colour intensity. 


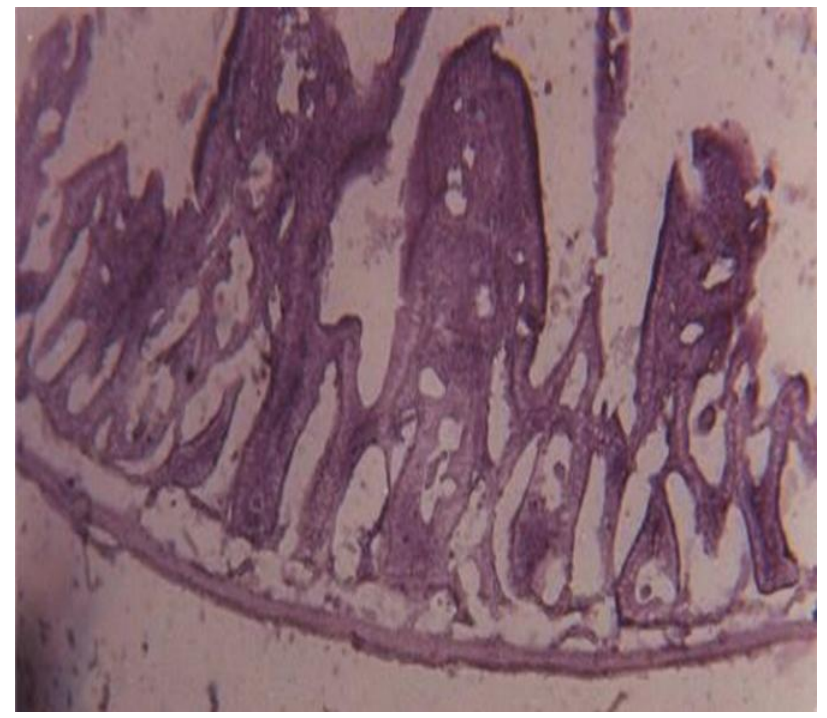

Fig.2c: Nitroblue tetrazolium stained intestine section of praziquantel treated mouse infected with H.nana, showing succinate dehydrogenase activity as that of control section.

\section{Conclusion}

The inferences drawn from the present work may serve as the preamble for further research in combating hymenolepiasis infection for the development of new prophylactic anthelmintics. This is needed because, in the tropics and subtropics, multiple worm infections are common[30-31]. If such periodical measures are observed, the mental and physical growth of the children especially in such colonies can be boosted. Moreover, in these countries, there is a need to develop many such cost-effective, safe, single, low-dosage regimen drugs, which can be administered orally without specific diet requirements[32-33]. The research can still further be extended for the development of vaccines[34].

\section{Acknowledgements}

We are grateful to the Management of Vivek Vardhini Society for permitting us to utilize the cryostat, microtome and photomicroscope of the P.G. Research laboratory of the Department of Zoology, at Vivek Vardhini College, Hyderabad.

\section{REFERENCES}

[1] D.L. Belding, Textbook of Parasitology, (New York: Appleton-Century-Crafts,1965) 1374.

[2] R.A. Wardle, J.A.Mc Leod, The Zoology of Tapeworms, (Minnesota: Univ. of Minnesota Press, 1952).

[3] T.C. Cheng, The biology of animal parasites, (Philadelphia: W.B. Saunders, 1964) 727.

[4] J. Parvathi, and K. Aruna, Histopathological assay of induced hymenolepiasis in Mus musulus and restoration of normalcy with praziquantel, The Bioscan , 5(4), 2010, 661-664.

[5] K.D. Murrell, Helminths, The Mouse in Biomedical Research. Experimental Biology and Oncology. Vol. IV (Academic Press, 1982) 225.

[6] P.A. Marks, and P. Feigelson, The biosynthesis of nucleic acid, ribose and of glycogen, glucose in rat, J Biol Chem , 226, 1957, 1001-1010.

[7] S.G. Arakcheeva, M.A. Zubitskaya, and K.H.B. Isaeva, Comparitive histochemical analysis of nuclei acids, carbohydrates and alkaline phosphatase in Hymenolepis nana and in the host tissues, Medit Parazit I Pazit Bolezni, 45(1), 1976, 81-84.

[8] J.K. Srivastava, Suman Gupta, and J.C. Katiyar, Effects of CDRI compounds on energy metabolism of A.ceylanicum and N. brasiliensis, Ind J Exptl Biol, 27(8), 1988, 735-738.

[9] S.P. Singh, J.C. Katiyar, and V.M.L. Srivastava, The enzymes of the tricarboxylic acid cycle in Ancylostoma ceylanicum and Nippostrongylus brasiliensis, J Parasitol, 78(1), 1992, 24-29.

[10] W. Schiebell, and H.J. Saz, The pathway for anaerobic carbohydrate dissimilation in Hymenolepis diminuta, Comp Biochem Physiol, 18, 1966, 151-162.

[11] J. Barrett, Biochemistry of parasitic helminthes, (London: McMillan Publ Ltd, 1981a) 283-286.

[12] J. Barrett, Biochemistry of parasitic helminthes, (London: McMillan Publ Ltd,1981b) 130. 
[13] E. Bueding, Effects of drugs on metabolism and enzyme systems of parasites, J Parasitol, 36, 1950, 201240.

[14] H. Thomas, and R. Gonnert, The efficacy of praziquantel against cestodes in animals, Zeit Fur Paras Parasitol Res, 52(2), 1977, 117-127.

[15] Lissau Bodil. Good Laboratory Practice, Handbook of Laboratory Animal Science: Selection and Handling of animals in Biomedical Research, Vol I (London, NewYork: CRC Press, 1994) 37-40.

[16] A.G.E. Pearse, Histochemistry:Theoretical and Applied, Analytical technology, Vol II (London, New York: Churchill Livingstone, 1985).

[17] B.E.H. Sumner, Basic Histochemistry, (John Wiley and Sons, 1988).

[18] J.F.A. Mc Manus, Histological demonstration of mucin after periodic acid, Nature, 158, 1946, 202.

[19] G. Gomori, Histochemical methods for protein-bound sulphhydryl and disulphide groups, Quart J Micr Sci, 97, 1956, 1 .

[20] R.D. Lillie, Histopathologic technic and practical histochemistry, (Mc Graw-Hill, 1965).

[21] A.G.E. Pearse, Application of the alkaline tetrazolium reaction to the study of reducing groups in tissue sections, J Pathol Bact, 67, 1954, 129.

[22] L.W. Roberts, and G. Lucchese, Sulfhydryl localization and tetrazolium reduction. 1.Reversible inhibition of its reduction by N-ethyl maleimide, Stain Technol, 30,1955, 291.

[23] K.C. Tsou, C.S. Chang, M.M. Nachlas, and A.M. Seligman, Synthesis of some $\rho$-nitrophenyl substituted tetrazolium salts as electron acceptors for the demonstration of dehydrogenases, J Amer Chem Soc, 78,1956, 6139 .

[24] J.D. Bancroft, Enzyme histochemistry, In Bancroft, JD, Stevens A (Ed.), Theory and practice of histological techniques, (Churchill Livingstone, 1966) 407.

[25] J. Parvathi, and K. Aruna, Leucocyte Variation, an Insight of Host Defenses During Hymenolepiasis and Restoration with Praziquantel, Ind J Pharma Sci, 73(1), 2011, 76-79.

[26] H. Selye, Effect of muscular exercise on the fat content of liver, Anat Rec, 73, 1939, 391.

[27] L.S. Chappell, Physiology of parasites, (London: Blackie, 1980).

[28] P. Andrews, Pharmacokinetic studies with Drocit in animals using a biological assay, Vet Med Nachr, 2,1976, 154-165.

[29] P. Andrews, and H. Thomas, The effect of praziquantel on Hymenolepis diminuta in vitro, Trop und Parasitologie, 30, 1979, 391-400.

[30] D.M. Cook, R.C. Swanson, D.L. Eggett, G.M. Booth, A retrospective analysis of prevalence of gastrointestinal parasites among school children in the Palajunoj Valley of Guatemala, J Health Popul Nutr, 27(1), [Medline], 2009, 31-40.

[31] M.A. Staat, M. Rice, S. Donauer, S. Mukkada, M. Holloway, and A. Cassedy, Intestinal Parasite Screening in Internationally Adopted Children: Importance of Multiple Stool Specimens, Pediatrics, [Medline], 2011.

[32] J.J. Ortiz, L. Favennec, N.L. Chegne, and G. Gargala, Comparative clinical studies of nitazoxanide, albendazole and praziquantel in the treatment of ascariasis, trichuriasis, and hymenolepiasis in children from Peru, Trans Royal Soc Trop Med Hyg, 96, 2002, 193-196.

[33] U.E. Meltem, G. Aynur, and A. Handan, Efficacy of praziquantel (injection formula) in the treatment of Hymenolepis diminuta infection in laboratory rats by oral application, Trop Med and Health, Online, 2009,1349-4147.

[34] M.U. Ahmed, D.P. Banerjee, and O.P. Gautam, Vaccination against Ancylostoma caninum in dogs. III. Polyacrylamide gel electrophoresis of serum proteins following vaccination with irradiated larvae. Ind J Parasitol, 10(2), 1986, 277-281. 